\title{
OPEN SETS OF POINTS WITH GOOD STABILIZERS
}

\author{
BY FRANK GROSSHANS ${ }^{1}$
}

Communicated by Hyman Bass, September 22, 1973

1. Observable groups and invariant theory [2]. Throughout this paper, $k$ denotes a fixed algebraically closed field of characteristic 0 and $G$ a connected, reductive algebraic group defined over $k$. (Our definitions and theorems regarding algebraic groups are taken from [1]. From now on, we shall always write variety instead of $k$-variety and morphism instead of $k$ morphism. A variety will always be identified with its $k$-rational points.) The subgroups of $G$ in which we are interested are described in the next two definitions.

An algebraic subgroup $H$ of $G$ is called observable in $G$ if there is a finite-dimensional rational representation $\rho: G \rightarrow G L(V)$ and a vector $v \in V$ such that $H=S_{G}(\rho, v)=\{g \in G \mid \rho(g) v=v\}$.

An observable subgroup $H$ of $G$ is said to satisfy the codimension 2 condition on $G / H$ if there is a finite-dimensional rational representation $\rho: G \rightarrow G L(V)$ and a vector $v \in V$ such that

(a) $H=S_{G}(\rho, v)$;

(b) each irreducible component of $\mathrm{Cl}(G \cdot v)-G \cdot v$ has codimension $\geqq 2$ in $\mathrm{Cl}(G \cdot v)$.

(In (b), $G \cdot v$ denotes the orbit of $v$ under the action of $G$. Furthermore, if $A$ is a subset of some affine space $k^{m}$, we shall always denote by $\mathrm{Cl}(A)$ the Zariski-closure of $A$ in $k^{m}$.)

Now let $Z$ be an irreducible affine variety and let $k[Z]$ be the ring of regular functions on $Z$. We assume that $G$ operates regularly on $Z$ via a mapping from $G \times Z \rightarrow Z$ denoted by $(g, z) \rightarrow g \cdot z$. Then $G$ operates on $k[Z]$ as follows: $(g \cdot f)(z)=f\left(g^{-1} \cdot z\right)$ for all $f \in k[Z], z \in Z$, and $g \in G$. For $H$ any subgroup of $G$ we put $k[Z]^{H}=\{f \in k[Z] \mid h \cdot f=f$ for all $h \in H\}$.

The importance of observable groups in invariant theory is illustrated by the two following results.

(i) Given any subgroup $H$ of $G$, there is an observable subgroup $H^{\prime \prime}$ of $G$ such that for any irreducible affine variety $Z$ on which $G$ operates regularly, we have $k[Z]^{H}=k[Z]^{H^{\prime \prime}}$.

AMS (MOS) subject classifications (1970). Primary 20G05.

Key words and phrases. Orbit, invariants.

${ }^{1}$ This research has been partially supported by the National Science Foundation. 
(ii) For $H$ an observable subgroup of $G$, the following conditions are equivalent:

(a) $H$ satisfies the codimension 2 condition on $G / H$;

(b) if $Z$ is any irreducible affine variety on which $G$ operates regularly, then $k[Z]^{H}$ is a finitley generated ring over $k$.

Again, let $G$ operate regularly on an irreducible affine variety $Z$. We say that $(Z, G)$ satisfies condition $(C)$ if there is a nonempty, $G$-invariant, Zariski-open set $U$ in $Z$ such that for each $u \in U$, the stabilizer of $u$ in $G$, denoted by $S_{G}(u)$, satisfies the codimension 2 condition on $G / S_{G}(u)$. (Incidentally, this does not necessarily mean that if $u \in U$, then each irreducible component of $\mathrm{Cl}(G \cdot u)-G \cdot u$ has codimension $\geqq 2$ in $\mathrm{Cl}(G \cdot u)$.) The purpose of this paper is to prove the following:

THEOREM. If $\rho: G \rightarrow G L(V)$ is a finite-dimensional rational representation of $G$, then $(V, G)$ satisfies condition $(\mathrm{C})$.

Our proof is derived from a method of $\mathbf{J}$. Kozul in the theory of compact transformation groups which allows an induction procedure to work. Recently, this method has been described and used in an algebraic group setting by D. Luna and R. Richardson [4], [6]. In particular, our proof of the theorem above is the same as Richardson's proof of the existence of principal isotropy subalgebras with a small modification and the notable use of the following results from [2].

1. An algebraic subgroup $H$ of $G$ is observable in $G$ if and only if $H_{0}$ is. In this case, $H$ satisfies the codimension 2 condition on $G / H$ if and only if $H_{0}$ does [2, $\S 3$, Remark (ii), and $\S \S 5,3$, Remark C].

2. Let $H$ be an observable subgroup of $G$. If there is a finite-dimensional rational representation $\rho: G \rightarrow G L(V)$ and a vector $v \in V$ such that $H=$ $S_{G}(\rho, v)$ and $k[\mathrm{Cl}(G \cdot v)]$ is a unique factorization domain, then $H$ satisfies the codimension 2 condition on $G / H[2, \S \S 5,3$, Remark A].

3 . Let $G$ be a connected, reductive algebraic subgroup of some $G L_{n}(k)$ and let $H$ be an algebraic subgroup of $G$. Then $H$ is observable in $G$ if and only if $H$ is observable in $G L_{n}(k)$. Furthermore, in this case, $H$ satisfies the codimension 2 condition on $G / H$ if and only if $H$ satisfies the codimension 2 condition on $G L_{n}(k) / H[2, \S 6$, Theorem 4].

2. A quotient variety [4], [6]. In this section, $H$ is a connected reductive subgroup of $G$. Let $H$ operate regularly on the irreducible affine variety $X$ via a mapping from $H \times X \rightarrow X$ denoted by $(h, x) \rightarrow h \cdot x$. Then $H$ operates regularly on $G \times X$ as follows: $h \cdot(g, x)=\left(g h^{-1}, h \cdot x\right)$. Since the stabilizer in $H$ of each point in $G \times X$ is $\{e\}$, all the orbits have the same dimension and so are closed. It follows that the quotient variety $(G \times X) / H$ exists and is affine with $k[(G \times X) / H]=k[G \times X]^{H}[5$, Chapter 1 , 2 , Amplification 
1.3]. We shall let $\phi: G \times X \rightarrow(G \times X) / H$ be the canonical map and shall write $\phi:(g, x) \rightarrow[g, x]$. The group $G$ operates regularly on $(G \times X) / H$ by $g^{\prime} \cdot[g, x]=\left[g^{\prime} g, x\right]$. Furthermore, the relationship between the stabilizers in $H$ of points in $X$ and the stabilizers in $G$ of points in $(G \times X) / H$ is given by

$$
S_{G}([g, x])=g S_{H}(x) g^{-1} .
$$

An important fact about $(G \times X) / H$ not explicitly stated before is the next

Lemma. The canonical mapping $\phi: G \times X \rightarrow(G \times X) / H$ is flat.

Proof. We need to show that the mapping $\Psi: H \times(G \times X) \rightarrow(G \times X) \times$ $(G \times X)$ given by $\Psi(h,(g, x))=\left(g h^{-1}, h \cdot x\right) \times(g, x)$ is a closed immersion [5, Proposition 0.9, p. 16, and Definition 0.8, p. 9]. Let $Z=G \times X$ and let $k[Z]^{H}=k\left[f_{1}, \cdots, f_{r}\right]$. Then the image of $\Psi$ is $\left\{(a, b) \in Z \times Z \mid f_{i}(a)=\right.$ $f_{i}(b)$ for each $\left.i=1, \cdots, r\right\}$; this closed set is isomorphic to $H \times(G \times X)$ and the Lemma is proved.

Corollary. If $X$ is regular, then so is $(G \times X) / H$.

Proof. This follows at once from [3, Chapter 4, $\S 6$, Corollary 6.5.2].

3. Proof of Theorem [6]. We need two preliminary results.

1. Let $\rho: G \rightarrow G L(V)$ be a finite-dimensional rational representation of $G$ and assume that there does not exist an open $G$-orbit on $V$. Then there is a vector $v \in V, v \neq 0$, such that $S_{G}(\rho, v)$ is reductive [6, Lemma 1.1].

2. Let $G$ act regularly on the irreducible affine varieties $X$ and $Y$ and let $\alpha: X \rightarrow Y$ be a $G$-equivariant morphism. Assume that there is a simple point $x \in X$ such that $\alpha(x)$ is a simple point of $Y$ and the differential $(d \alpha)_{x}$ is a linear isomorphism. Then if $(X, G)$ satisfies condition (C), so does $(Y, G)[6$, Lemma 3.1].

Proof. Under the assumptions above, Richardson shows that there is an open $G$-invariant subset $U$ in $X$ containing $x$ such that $\alpha(U)$ is an open, $G$-invariant subset of $Y, \alpha \mid U$ is an open map, and $S_{G}(u)_{0}=S_{G}(\alpha(u))_{0}$ for each $u$ in $U$. Our conclusion now follows from 1 in $\S 1$.

Proof OF THEOREM. We proceed by induction on $(\operatorname{dim} V+\operatorname{dim} G)$, the case where $\operatorname{dim} V+\operatorname{dim} G=0$ being trivial. Now if $G$ has an open orbit on $V$, then $(V, G)$ satisfies condition (C) by 2 in $\S 1$. Also, if $V^{a}=$ $\{v \in V \mid \rho(g) v=v\}$ is not $\{0\}$, then there is a $G$-invariant subspace $V_{0}$ of $V$ such that $V=V_{0} \oplus V^{G}$; in this case, we have that $\left(V_{0}, G\right)$ satisfies condition (C) by induction and, hence, so does $(V, G)$. From now on, then, we may assume that $G$ has no open orbit on $V$ and that $V^{G}=\{0\}$.

By 1 above, there is a vector $v \in V, v \neq 0$, such that $S_{G}(\rho, v)$ is reductive. We let $H=S_{G}(\rho, v)_{0}$; by our assumptions $\operatorname{dim} H<\operatorname{dim} G$. Now, if we 
denote by $T_{v}$ the tangent space to $G \cdot v$ at $v$ and identify $T_{v}$ with an $H$ invariant subspace of $V$, then we may find an $H$-invariant subspace $W$ of $V$ such that $V=W \oplus T_{v}$. Using $W$, we construct the quotient space $(G \times W) / H$ as in $\S 2$ and let $\Phi: G \times W \rightarrow(G \times W) / H$ be the canonical map.

By induction, $(W, H)$ satisfies condition (C). Hence, there is a nonempty, $H$-invariant, Zariski-open set $U_{1}$ in $W$ such that, for each $u_{1}$ in $U_{1}$, $S_{H}\left(u_{1}\right)$ satisfies the codimension 2 condition on $H / S_{H}\left(u_{1}\right)$. Then, $U=$ $\phi\left(G \times U_{1}\right)$ is a nonempty, $G$-invariant, Zariski-open subset of $(G \times W) / H$ such that, for each $u$ in $U, S_{G}(u)$ satisfies the codimension 2 condition on $G / S_{G}(u)$. Indeed, if $u=[g, w]$, then we have seen that $S_{G}(u)=g S_{H}(w) g^{-1}$; but $S_{H}(w)$ satisfies the codimension 2 condition on $H / S_{H}(w)$, therefore, on $G / S_{H}(w)$ by 3 in $\S 1$.

Finally, let $\tau: G \times W \rightarrow V$ be defined by $\tau(g, w)=g \cdot(v+w)$; it is easy to check that $(d \tau)_{(e, 0)}$ is onto. There is a mapping $\psi:(G \times W) / H \rightarrow V$ such that $\tau=\psi \circ \phi$. Furthermore, $\psi$ is a $G$-morphism and (since $(G \times W) / H$ is regular) $(d \psi)_{[e, 0]}$ is a linear isomorphism. The Theorem now follows from 2 above.

\section{BIBLIOGRAPHY}

1. A. Borel, Linear algebraic groups, Benjamin, New York, 1969. MR 40 \#4273.

2. F. Grosshans, Observable groups and Hilbert's fourteenth problem, Amer. J. Math. (1973) No. 195.

3. A. Grothendieck, Elements de géométrie algébrique (with J. Dieudonné), Inst. des Hautes Scientifiques, Paris, 1965.

4. D. Luna, Sur les orbites fermées des groupes algébriques réductifs, Invent. Math. 16 (1972), 1-5. MR 45 \#3421.

5. D. Mumford, Geometric invariant theory, Ergebnisse der Math. und ihrer Grenzgebiete, Neue Folge, Band 34, Springer-Verlag, Berlin and New York, 1965. MR 35 \#5451.

6. R. W. Richardson, Jr., Principal orbit types for algebraic transformation spaces in characteristic zero, Invent. Math. 16 (1972), 6-14. MR 45 \#3405.

Department of Mathematics, Johns Hopkins University, Baltimore, Maryland 21218 